\title{
INTRODUCGIÓN AL ESTUDIO DEL ESTATUS EPISTEMOLÓGICO DE LAS CIENCIAS ADMINISTRATIVAS EN MARIO BUNGE
}

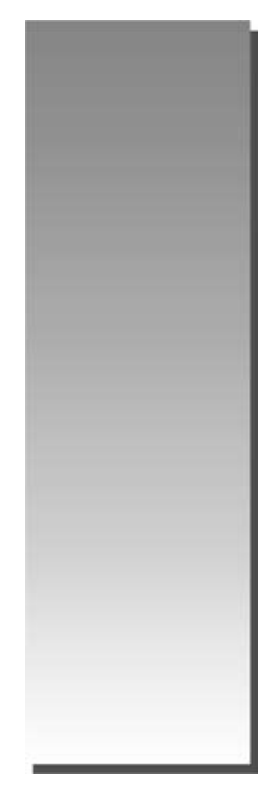

Julio López Mas (")

E-mail: johans_jlopez@yahoo.com

\begin{abstract}
RESUMEN
Las ciencias administrativas tienen como objeto de estudio las organizaciones que se desarrollan a través de la toma de decisiones y, aunque se auxilian en diversas ciencias y técnicas, mantienen un carácter específico, demostrando unidad temporal y jerárquica, valor instrumental, amplitud, interdisciplinariedad y flexibilidad. El artículo analiza críticamente la posición de Bunge sobre la naturaleza epistemológica de las ciencias administrativas.
\end{abstract}

Palabras clave: Epistemología, Mario Bunge, Ciencias Administrativas.

\section{ABSTRACT}

The administrative sciences have as object of study the organizations, that develop through decisions taken and, although they are helped in diverse sciences and technical's, it maintains a specific character, showing hierarchical and temporary unit, instrumental value, amplitude, interdisciplinary nature and flexibility. The article analyzes critically the position of Bunge on the epistemological nature of the administrative sciences.

Key words: Epistemology, Mario Bunge, Administrative Science.

(*) Doctor de las Economías y Sociedades Latinoamericanas (EHESS, París). Profesor Principal y Director de Instituto de Investigaciones de la Facultad de Ciencias Administrativas de la UNMSM. 


\section{CIENCIA, TECNOLOGÍA Y SOCIEDAD}

Hoy en día las cuestiones relativas a la ciencia y la tecnología, y su importancia en la definición de las condiciones de la vida humana, desbordan el ámbito académico para convertirse en centros de interés del conjunto de la sociedad globalizada.

Ciencia, tecnología y sociedad configuran una tríada más compleja que una simple serie sucesiva, y su combinación obliga a analizar sus relaciones recíprocas. Los estudios sobre ciencia, tecnología y sociedad requieren de un análisis crítico e interdisciplinario, así como la caracterización de su contexto social.

La revolución científica de los siglos XVII y XVIII no sólo fue de orden teórico, conceptual y metodológico, sino que sacudió al mundo con sus transformaciones sociales que hasta hace poco considerábamos impresionantes, pero que comienzan a palidecer ante lo que ocurre en la sociedad actual.

Las fronteras del conocimiento se han desbordado, o más bien parecen ya no tener límite: el conocimiento ha abierto posibilidades de intervención en cuanta esfera de la vida humana y de la naturaleza nos podamos imaginar: de las comunicaciones a la actividad mental, del genoma humano a la exploración del espacio, de la procreación a la carta a formas sin precedente de invadir la privacidad de las personas ${ }^{1}$.

El vertiginoso desarrollo científico-tecnológico del mundo actual está consiguiendo hacer realidad lo que hasta hace sólo unas décadas eran fantasías, con un extraordinario potencial para la transformación de la naturaleza y la satisfacción de las necesidades humanas. Este nuevo milenio -caracterizado por la creciente globalización en el ámbito tecno-económico (basada en la energía nuclear, la biotecnología y las tecnologías de la información), y también por la acentuación de las diferencias en la distribución del conocimiento y de la riqueza- es un mundo de beneficios y amenazas globales, pero también de profundas asimetrías en la distribución de la riqueza, los costes ambientales y la apropiación del conocimiento científico.

\section{PROBLEMA}

La administración de empresas, ciencia administrativa o simplemente administración, es una ciencia social que estudia la organización de las empresas y la manera cómo se gestionan los recursos, procesos y resultados de sus actividades.

Se dice que administrar es planear, organizar, dirigir y controlar todos los recursos de un ente económico para alcanzar unos fines claramente determinados. Se apoya en otras ciencias como la economía, sociología, psicología y antropología para ejercer sus funciones.

El fenómeno administrativo se da donde quiera que exista un organismo social. Es el proceso global de toma de decisiones orientado a conseguir los objetivos organizativos de forma eficaz y eficiente mediante la planificación, organización, integración de personal, dirección (liderazgo) y control. Es una ciencia que se basa en técnicas viendo a futuro, coordinando cosas, personas y sistemas para lograr, por medio de la comparación y jerarquía, un objetivo con eficacia y eficiencia. La toma de decisiones es la principal fuente de una organización para llevar a cabo buenas actividades, coordinación sistemática de medios y obtener excelentes resultados.

La administración se realiza en el Estado, en el ejército, en la empresa, en las instituciones educativas, en una sociedad religiosa, etc., y los elementos esenciales en todas esas clases de administración serán los mismos, aunque lógicamente existen variantes accidentales.

- Universalidad. Se puede decir que la administración es universal porque se puede aplicar en todo tipo de organismo social y en todos los sistemas políticos existentes.

- Especificidad. Aunque la administración va siempre acompañada de otros fenómenos de índole distinta, el fenómeno administrativo es específico y distinto a los que acompaña. Se puede ser un magnífico ingeniero de producción y un pésimo administrador. La administración tiene características específicas que nos impiden confundirla con otra ciencia o técnica. Aunque la administración se auxilia de otras ciencias y técnicas, tiene características propias que le proporcionan su carácter específico; es decir, no puede confundirse con otras disciplinas.

- Unidad temporal. Aunque se distingan etapas, fases y elementos del fenómeno administrativo, éste es único y, por lo mismo, en todo

1 Oliv, León. (2007). Pág. 13. 
momento de la vida de una empresa se están dando, en mayor o menor grado, todos o la mayor parte de los elementos administrativos. Así, al hacer los planes, no por eso se deja de mandar, de controlar, de organizar, etc.

- Unidad jerárquica. Todos los que tienen carácter de jefe en un organismo social participan en distintos grados y modalidades de la misma administración. Así, en una empresa forman un solo cuerpo administrativo el gerente general hasta el último operario.

- Valor instrumental. La administración es un medio para alcanzar un fin; es decir, se utiliza en los organismos sociales para lograr en forma eficiente los objetivos establecidos.

- Amplitud de ejercicio. Se aplica en todos los niveles de un organismo formal, por ejemplo, presidentes, gerentes, supervisores, ama de casa, etc.

- Interdisciplinariedad. La administración hace uso de los principios, procesos, procedimientos y métodos de otras ciencias y técnicas que están relacionadas con la eficiencia en el trabajo. Está relacionada con matemáticas, estadística, derecho, economía, contabilidad, sociología, psicología, filosofía, antropología, etc.

- Flexibilidad. Los principios y técnicas administrativas se pueden adaptar a las diferentes necesidades de la empresa o grupo social.

Sin embargo, en la actualidad, existen puntos de vista que niegan o relativizan el carácter científico de la administración, pese a que la administración es una carrera profesional en boga y hay un continuo cambio en las organizaciones y empresas, además que en muchos foros de líderes del mundo académico y empresarial se estudian y perfeccionan enfoques y prácticas de la administración y del management del futuro, con nuevas perspectivas para el diseño de organizaciones más adaptables, innovadoras y humanas.

Uno de los pensadores con gran renombre e influencia en la comunidad científica es Mario Bunge. Este filósofo, al hablar de la administración, dice:

"Si la administración se considera como una ciencia (social) entonces su objetivo central debe ser el de buscar leyes y normas que satisfagan o debieran satisfacer la actividad administrativa, desde el contador hasta el gerente de producción y el encargado de relaciones públicas. En cambio, si la administración es una tecnología, dejará esa investigación básica a las ciencias sociales puras, para ocuparse entonces de diseñar modelos de organización óptima sobre la base de conocimientos adquiridos en las ciencias básicas, así como de nuevos conocimientos adquiridos en el curso de la investigación y la experiencia administrativa"2.

La epistemología, o filosofía de la ciencia es la rama de la filosofía que estudia la investigación científica y su producto, que es el conocimiento científico. Su importancia actual radica en que la ciencia se ha convertido en el eje de la cultura contemporánea y en un motor de la sociedad del conocimiento.

“... y, por ser el motor de la tecnología, la ciencia ha venido a controlar indirectamente la economía de los países desarrollados. Por consiguiente, si se quiere adquirir una idea adecuada de la sociedad moderna, es menester estudiar el mecanismo de la producción científica, así como la naturaleza de sus productos"3.

El presente artículo se plantea como objetivo realizar un análisis crítico de esta posición de Mario Bunge; sin embargo, las interrogantes planteadas no sólo se refieren a la relación entre las ciencias sociales y la tecnología social en el pensamiento de Bunge, también nos interesa realizar una comprensión de la discusión filosófica y epistemológica actual sobre las ciencias en general y las ciencias sociales en particular. Este esclarecimiento conceptual es bastante relevante con nuestro objetivo ya que sabemos que la epistemología participa del desarrollo científico al contribuir a cambiar positivamente el trasfondo filosófico de la investigación así como de la política de la ciencia, y también nos ayuda a la construcción de puentes entre las disciplinas.

\section{MARIO BUNGE Y LA FILOSOFÍA DE LA CIENCIAS SOCIALES}

La filosofía de la ciencia es la investigación sobre la naturaleza del conocimiento científico

2 Bunge, Mario. (1993). Pág. 53.

3 Bunge, Mario. (2006a). Pág. 17. 
y la práctica científica. Se ocupa de saber, entre otras cosas, cómo se desarrollan, evalúan y cambian las teorías científicas, y de saber si la ciencia es capaz de revelar la verdad de las "entidades ocultas" (o sea, no observables) y los procesos de la naturaleza.

Bunge dice:

“... Creo que el deber de los filósofos es tratar de resolver problemas conceptuales difíiles e interesantes, cuyas soluciones pueden ser de utilidad para otros pensadores y para hombres y mujeres de acción de todo tipo. En particular, creo que el filósofo de las ciencias sociales tiene el deber de dilucidar los conceptos claves, los problemas, los supuestos tanto ocultos como explícitos- y los descubrimientos sociales de su época"'.

La filosofía de la ciencia no se denominó así hasta la formación del Círculo de Viena, a principios del siglo XX. En la misma época, la ciencia vivió una gran transformación a raíz de la teoría de la relatividad y de la mecánica cuántica. En la filosofía de la ciencia actual las figuras más conocidas son Karl R. Popper, Thomas Kuhn, Imre Lakatos y Paul Feyerabend.

Es extremadamente complejo presentar un panorama completo de la filosofía de la ciencia de los últimos treinta o treinta y cinco años y ello tampoco es el propósito de nuestra investigación. Así como anteriormente se podía hablar de "el método" de la ciencia, el gran desarrollo de muchas disciplinas científicas ha hecho que los filósofos de la ciencia comiencen a hablar de "los métodos", ya que no es posible identificar un método único y universalmente válido. La idea heredada de la física clásica de que todo es reducible a expresiones matemáticas ha cedido terreno ante situaciones nuevas como la teoría del caos, los avances de la biología o las tecnologías informáticas.

El realismo crítico es la creencia que la realidad, existiendo y siendo objetiva, no puede ser conocida de manera absoluta, siendo nuestro conocimiento del mundo aproximativo. No es posible la certeza, nunca se puede saber cuándo nuestro conocimiento es cierto. Con orígenes claros en Kant, ha sido defendido por Popper, Rom Harré, Roy Bhaskar, y Mario Bunge ${ }^{5}$, entre otros.

Karl Popper y Mario Bunge son defensores estrictos del realismo de la ciencia. Los realistas en sentido fuerte defienden que las teorías y conceptos científicos nos proporcionan conocimiento sobre la realidad porque hay algún tipo de relación de correspondencia entre las teorías científicas y la naturaleza. Mario Bunge analiza los problemas de diversas epistemologías desde el racionalismo crítico popperiano hasta el empirismo, el neopositivismo, el subjetivismo o el relativismo. Se considera un realista crítico que ve la ciencia como falible (el conocimiento del mundo es provisional e incierto), pero aún así afirma que la realidad existe y es objetiva. En el prefacio de 1997 de su obra "Epistemología" cuya primera edición es de 1980 nos dice: "Desde entonces (1980) he aprendido mucho, pero sigo siendo un realista, cientificista, materialista y sistemista convicto y confeso"6.

El realismo posee siete componentes: ontológico, gnoseológico, semántico, metodológico, axiológico, moral y praxeológico (concerniente a la teoría de la acción). Además, cada uno de sus constituyentes se presenta en diferentes matices: ingenuo, crítico y científico ${ }^{7}$.

4 Bunge, Mario. (2005). Pág. 10.

5 Dentro de la corriente racionalista de oposición al neopositivismo encontramos a Mario Bunge, un físico, filósofo, epistemólogo y humanista argentino. Nació en Buenos Aires el 21 de septiembre de 1919. Interesado en la filosofía de la física, Mario Bunge comenzó sus estudios en la Universidad Nacional de La Plata, graduándose con un doctorado en ciencias físico-matemáticas en 1952. En la Universidad de Buenos Aires fue profesor de física teórica y filosofía desde 1956 hasta 1963 cuando, insatisfecho con el clima político de su país, tomó la decisión de emigrar.

Por unos pocos años Mario Bunge enseñó en universidades de México, Estados Unidos y Alemania. Finalmente, en 1966 se instaló en Montreal (Canadá), donde enseña en la Universidad McGill desde entonces, ocupando la cátedra Frothingam de lógica y metafísica.

Mario Bunge es autor de más de medio millar de monografías y una cincuentena de libros de física y filosofía, entre ellos "Treatise on Basic Phylosophy", "Las ciencias sociales en discusión”, "Diccionario de filosofía”, "A la caza de la realidad" y "Filosofía y sociedad". Acaba de aparecer en español el primero de los ocho tomos de su "Tratado de Filosofía". Ha sido honrado en varias ocasiones con doctorados honoris causa otorgados por instituciones como la Universidad de Salamanca (España) en 2003, la Universidad Nacional de La Plata (Argentina) y la Universidad de Buenos Aires (Argentina) en 2008. También recibió el Premio Príncipe de Asturias en 1982.

6 Bunge, Mario. (2006a). Pág.13.

7 Bunge, Mario. (2007a). Pág. 57. 


\section{CONCEPTOS BÁSICOS: CIENCIAS SOCIALES Y TÉCNICAS SOCIALES}

Para Mario Bunge ciencia es la búsqueda crítica de pautas o la utilización de éstas en las ideas, la naturaleza o la sociedad, y puede ser formal o factual. Es formal si se refiere a los constructos -conceptos, proposiciones, clasificaciones, teorías, (por ejemplo la lógica y las matemáticas)- y factual si trata cuestiones de hechos (la física y la historia, pero también todas las ciencias que existen entre ambas son factuales porque tratan de cosas concretas tales como rayos de luz o empresas comerciales). Las ciencias factuales pueden dividirse en naturales (por ejemplo, la biología), sociales (por ejemplo, la economía, que es la rama de las ciencias sociales que estudia los sistemas económicos como las empresas, las industrias, los mercados y las economías globales) y biosociales (por ejemplo, la psicología) ${ }^{8}$.

Pero la ciencia no es simplemente un cuerpo de conocimientos; es asimismo una actividad de investigación que se da en una sociedad en el curso de la historia. Es decir, el concepto de ciencia comprende distintos aspectos: conceptual, empírico, social e histórico ${ }^{9}$, pero esta división de las ciencias no nos hace perder la comprensión del carácter sistémico del conocimiento humano (todo hecho social involucra agentes que actúan en algunos sistemas sociales; todos los sistemas sociales cambian constantemente, y los hechos deben ser explicados de un modo objetivo).

A diferencia del enfoque fragmentario que estudia o manipula cosas fuera de sus contextos, el enfoque sistémico presta atención al contexto. Para Mario Bunge:

“... toda ciencia es un sistema conceptual y es, a su vez, un miembro del sistema del conocimiento humano. Ninguna ciencia fáctica es independiente o autosuficiente: toda disciplina interactúa con otras disciplinas y todas ellas poseen un núcleo lógico, matemático y filosófico común"10.

Aplicado a la sociedad -señala Mario Bunge en el Prólogo de su obra "Sistemas Sociales y
Filosofía"11- el enfoque sistémico es caracterizado como un sistema extremadamente complejo, compuesto por subsistemas interactuantes e interdependientes tales como la familia, la empresa, la escuela, el club, la administración pública y el partido político. Estos sistemas están compuestos por individuos capaces de amar, odiar, imaginar, arguiir, evaluar, decidir y actuar. Son cualquier cosa menos entes pasivos o juguetes de los vendavales históricos pero, por estar ligados entre sí y por estar constreñidos por normas de diversas clases, las personas nunca son totalmente independientes y libres. Interactúan, y estas acciones mutuas hacen que los sistemas sociales sean interdependientes y dinámicos.

La concepción sistémica de la sociedad estimula la investigación de las propiedades y procesos peculiares de los sistemas sociales, así como la conducta social de sus miembros. Explica al individuo por la sociedad y a ésta por aquél. Por otro lado, sabiendo que la ciencia es un sistema tanto en el nivel conceptual como en el nivel social, el desarrollo científico debe ser multidisciplinario. No es posible desarrollar cualquiera de sus componentes sino se desarrollan los demás ${ }^{12}$.

Mario Bunge considera como las principales ramas de las ciencias sociales a la antropología, la sociología, la economía, la politología y la historia, cuyo objeto común es el estudio científico de los sistemas sociales y de la acción social. Las empresas, escuelas, gobierno, familia, comunidad religiosa y las redes sociales informales son sistemas sociales (sistema cuyos componentes son seres humanos). En líneas generales, la acción humana es cualquier cosa que hacen los humanos aunque Mario Bunge define la teoría de la acción como praxiología ${ }^{13}$.

Para el propósito de nuestra investigación es importante establecer la definición de sistema técnico y tecnología social.

- Sistema técnico es un sistema social en el que se emplea de modo destacado la tecnología avanzada. Ejemplos: las modernas plantas industriales, los ejércitos y los hospitales.

8 Bunge, Mario. (2006b). Págs. 22 y 57.

9 Bunge, Mario. (1988). Pág. 25.

10 Bunge, Mario. (2002). Págs. 6 y 59.

11 Bunge, Mario. (1999). Prólogo.

12 Bunge, Mario. (2006a). Pág. 236.

13 Bunge, Mario. (2006b). Págs. 24, 168 y 198. Junto con la ética, la praxiología es un componente de la filosofía práctica. 
- Tecnología es la rama del conocimiento que se ocupa de diseñar artefactos y procesos, así como de la normalización y el diseño de la acción humana. La tecnología moderna se basa en la ciencia y puede perfeccionarse mediante la investigación.

- Tecnología social es el diseño de políticas y planes para el mantenimiento, reparación o construcción de sistemas sociales, ya sean privados o públicos, partiendo de las ciencias sociales. Las principales ramas son: ciencia administrativa, gestión de recursos, macroeconomía normativa, trabajo social, jurisprudencia, criminología, demografía y planificación urbana ${ }^{14}$. La meta de los administradores es optimizar (no necesariamente maximizar) el desempeño técnico y económico de los sistemas socio-tecnológicos a su cargo ${ }^{15}$.

La distinción entre ciencia y técnica no implica separación alguna. Es una división del trabajo equivalente a las existentes en los pares verdad-eficiencia, desinterés-parcialidad y ciencia positiva-diseño de políticas. Es más, Mario Bunge nos señala la interacción entre ciencia y técnica en los siguientes términos:

“...muchos problemas de las ciencias sociales surgen debido a consideraciones prácticas; y a su vez, toda política o programa social eficiente surgirá y será guiado por algunos descubrimientos de la ciencia social básica"16.

Mario Bunge distingue la técnica de la tecnología: la primera es el resultado de una elaboración pre científica; la segunda es investigación y desarrollo basados en la ciencia.

La definición de tecnología comprende a todas las disciplinas orientadas a la práctica, siempre que practiquen el método científico. Mientras que el científico busca conocer por conocer, el tecnólogo busca conocer para hacer ${ }^{17}$, pero

"la diferencia principal entre la ciencia y la tecnología es que la primera, ya sea básica o aplicada, produce nuevos conocimientos, en tanto que los laboratorios, talleres y oficinas de investigación utilizan el conocimiento exclusivamente como un medio para diseñar artefactos (por ejemplo, máquinas, ciudades $u$ organizaciones formales), normas (por ejemplo, de emisión), o planes de acción (por ejemplo, campañas de alfabetización o de vacunación). Estas y otras peculiaridades de la tecnología son estudiadas por un número cada vez mayor de filósofos de la tecnología"18.

Los objetos centrales de la tecnología son artefactos, ya sean cosas o procesos, inanimados, vivos o sociales; y su meta es controlar los sistemas o procesos naturales o sociales para beneficiar a algún grupo social.

En su más reciente obra titulada "Filosofía y Sociedad"19, Mario Bunge sostiene que las ideas filosóficas valiosas no son fantasías ociosas, sino que guían tanto la investigación como la acción. Por ejemplo, un examen filosófico de la ingeniería muestra que es preferible adoptar la visión sistémica a la sectorial; $y$ un estudio de la filosofía del management puede promover estrategias eficaces $o$, al menos, desechar las ineficaces.

Las llamadas ciencias empresariales son necesariamente multidisciplinarias e interdisciplinarias porque tratan de organizaciones, y éstas tienen composiciones heterogéneas y estructuras cambiantes, y se encuentran sumergidas en entornos igualmente heterogéneos y cambiantes que tienen componentes económicos, políticos y culturales, y dependen críticamente del entorno ${ }^{20}$.

\section{BIBLIOGRAFÍA}

Bunge, Mario. (1988). Vigencia de la Filosofía: Ciencia y Técnica, Investigación y Universidad. Lima, Fondo Editorial de la Universidad Inca Garcilaso de la Vega.

Bunge, Mario. (1993). "Status epistemológico de la administración”, en J.J. Ader (Com) y otros. Organizaciones. Ciudad, Paidos, p. 52.

Bunge, Mario. (1999). Sistemas Sociales y Filosofía. Buenos Aires, Editorial Sudamérica.

14 Bunge, Mario (2006b). Op. cit. Págs. 198 y 199, 206 y 207.

15 Bunge, Mario. (2005). Op. cit. Pág. 289.

16 Bunge, Mario. (2002). Op. cit. Pág.129.

17 Bunge, Mario. (2006a). Op. cit. Págs. 191 y 197.

18 Bunge, Mario. (2005). Op. cit. Pág. 279.

19 Bunge, Mario. (2008).

20 Bunge, Mario. (2007b). Pág. 238. 
Bunge, Mario. (2002). Crisis y reconstrucción de la filosofía. Primera Edición. Barcelona, Editorial Gedisa.

Bunge, Mario. (2005). Buscar la filosofía en las ciencias sociales. Segunda Edición. México D.F., Siglo XXI Editores.

Bunge, Mario. (2006a). Epistemología. Quinta edición. México D.F., Siglo XXI Editores.

Bunge, Mario. (2006b). Diccionario de filosofía. Tercera edición. México D.F., Siglo XXI Editores.
Bunge, Mario. (2007a). A la caza de la realidad. La controversia sobre el realismo. Primera Edición. Barcelona, Editorial Gedisa.

Bunge, Mario. (2007b). Una filosofía realista para el nuevo milenio. Lima, Fondo Editorial de la Universidad Inca Garcilaso de la Vega.

Bunge, Mario. (2008). Filosofía y Sociedad. México D.F., Siglo XXI Editores.

Oliv, León. (2007). La ciencia y la tecnología en la sociedad del conocimiento. Primera edición en español. México D.F, Fondo de Cultura Económica. 